\title{
Stability and interactions of solitons in two-component active systems
}

\author{
Javid Atai ${ }^{1}$ and Boris A. Malomed ${ }^{2}$ \\ ${ }^{1}$ Department of Electrical Engineering, University of Twente, P.O. Box 217, 7500 AE Enschede, The Netherlands \\ ${ }^{2}$ Department of Applied Mathematics, School of Mathematical Sciences, Tel-Aviv University, Tel-Aviv 69978, Israel
}

(Received 27 March 1996; revised manuscript received 14 June 1996)

\begin{abstract}
We demonstrate that solitary pulses in linearly coupled nonlinear Schrödinger equations with gain in one mode and losses in another one, which is a model of an asymmetric erbium-doped nonlinear optical coupler, exist and are stable, as was recently predicted analytically. Next, we consider interactions between the pulses. The in-phase pulses attract each other and merge into a single one. Numerical and analytical consideration of the repulsive interaction between $\pi$-out-of-phase pulses reveals the existence of their robust pseudobound state, when a final separation between them takes an almost constant minimum value, as a function of the initial separation, $T_{\text {in }}$, in a certain interval of $T_{\text {in }}$. In the case of the phase difference $\pi / 2$, the interaction is also repulsive. [S1063-651X(96)12210-8]
\end{abstract}

PACS number(s): 42.81.Dp; 42.81.Qb; 52.35.Sb; 03.40.Kf

Localized pulses (solitons) play a central role in numerous physical systems that have attracted a lot of interest [1,2]. Real systems must contain an active element providing for a loss-compensating gain. In nonlinear optical fibers (NOF's) the losses can be compensated by the erbium-doped amplifiers [2]. However, if the active element is uniformly distributed in the system, it makes the zero solution unstable, thus lending instability to the solitary pulse. A well-known model with this property is the cubic Ginzburg-Landau (GL) equation [3]. In the application to NOF's, it may be regarded as a perturbed nonlinear Schrödinger (NLS) equation:

$$
i u_{z}+\frac{1}{2} u_{\tau \tau}+|u|^{2} u=i \gamma_{0} u+i \gamma_{1} u_{\tau \tau}-i \gamma_{2}|u|^{2} u
$$

where $u(z, \tau)$ is an envelope of the electromagnetic waves in the fiber, $z$ and $\tau$ are the propagation distance and the socalled reduced time, $\gamma_{0}$ is gain, and $\gamma_{1}$ and $\gamma_{2}$ are coefficients of the dispersive and nonlinear losses. Equation (1) has an exact solitary-pulse solution [3], which can form bound states [4]. However, at $\gamma_{0}>0$ the solution $u=0$ is unstable in this model, hence an isolated pulse is unstable too.

A problem of fundamental interest is to find a tractable physical model that can support stable pulses. Recently, it was proposed in [5] in the form of a dual-core NOF (coupler), in which one core is active while the other one is pure lossy. The model gives rise to two solitons, one stable and one unstable, the unstable one being a separatrix between attraction domains of the stable soliton and the stable zero solution.

In Ref. [5], only analytical results were presented. It remains necessary to check those results numerically, which is the first objective of the present work. It will be demonstrated that the pulses indeed exist and are stable. The shape of the numerically obtained pulses proves to be so close to the analytically predicted form that one virtually cannot distinguish between them. The other objective of this work is to consider collisions between the pulses. We will arrive at a simple concept of a "pseudo-bound state" of two pulses.
The model put forward in [5] is a system of two linearly coupled perturbed NLS equations for amplitudes of electromagnetic waves in an asymmetric twin-core NOF, only one core being active. Actually, one can have two identical doped cores, while the asymmetry is provided by pumping only one core using an external source of light. A coupler with one active core was first proposed in [6] to improve the quality of pulses generated by the soliton laser.

We will write the equations in the same "optical" notation as Eq. (1):

$$
\begin{gathered}
i u_{z}+\frac{1}{2} u_{\tau \tau}+|u|^{2} u-i \gamma_{0} u-i \gamma_{1} u_{\tau \tau}+\kappa v=0, \\
i v_{z}+\frac{1}{2} v_{\tau \tau}+|v|^{2} v+i \Gamma_{0} v+\kappa u=0
\end{gathered}
$$

Here, the variables $u$ and $v$ are the amplitudes of electromagnetic waves in the active and lossy cores of the coupler, $\gamma_{0}$ and $\gamma_{1}$ are the same as in Eq. (1), $\kappa$ is the coupling constant, and $\Gamma_{0}$ is the loss coefficient in the passive core.

First of all, one should consider the stability of the solution $u=v=0$. As was demonstrated in [5], a necessary and sufficient condition for its stability is

$$
\gamma_{0}<\Gamma_{0}<\kappa^{2} / \gamma_{0}
$$

In Ref. [5], the soliton in the model based on Eqs. (2) and (3) was treated perturbatively, assuming that the coupling, gain, and losses were small perturbations, although different perturbations were given different orders of smallness: the gain and losses in the active core were essentially weaker than the coupling between the cores, while the losses in the passive one might be comparable to the coupling.

In the zeroth-order approximation, the soliton is assumed to reside only in the first core:

$$
u=\eta \operatorname{sech}(\eta \tau) e^{i \phi(z)}, \quad v=0
$$




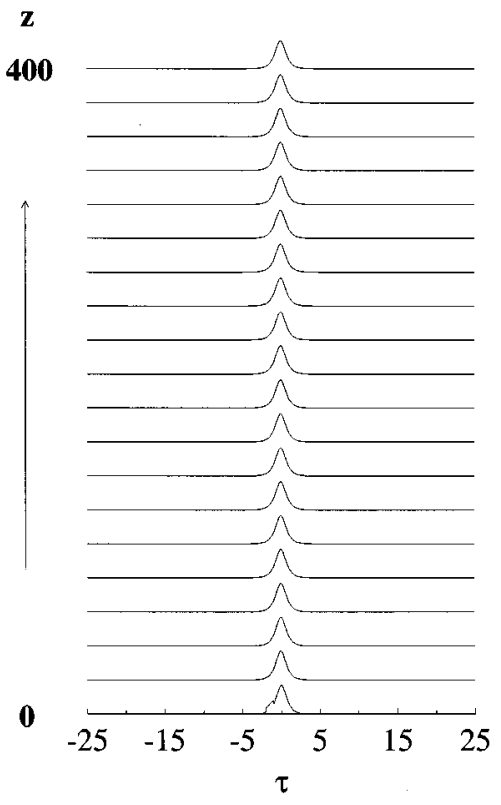

FIG. 1. An example of the numerical solution to Eqs. (2) and (3) with an initial state corresponding to a perturbed pulse: $|u(\tau)|$ is shown at different $z$.

where $(d \phi) /(d z)=\frac{1}{2} \eta^{2}$, and $\eta$ is the soliton's amplitude. In the next approximation, a component in the second core is sought as $v(z, \tau)=V(\tau) \exp [i \phi(z)]$, where the function $V(\tau)$ is determined by the equation

$$
\frac{d^{2} V}{d \tau^{2}}-\eta^{2} V=-2 \kappa \eta \operatorname{sech}(\eta \tau)
$$

which can be solved by the Fourier transform. Finally, an equation for slow evolution of the amplitude $\eta$ was derived in [5] by means of the balance equation for the energy:

$$
\frac{d \eta}{d z}=2 \gamma_{0} \eta-\frac{2}{3} \gamma_{1} \eta^{3}-C \kappa^{2} \Gamma_{0} \eta^{-3},
$$

where $C \equiv \frac{1}{6} \pi^{2}+\zeta(3) \approx 2.845$. The formal singularity of the last term in Eq. (7) at $\eta \rightarrow 0$ is fictitious, as this expression is irrelevant at very small $\eta$.

Equation (7) produces two physical $\left(\eta^{2}>0\right)$ fixed points provided that

$$
\gamma_{0}^{3}>(3 C / 8) \kappa^{2} \Gamma_{0} \gamma_{1}^{2},
$$

and no fixed point in the opposite case. Thus, (8) is the necessary and sufficient condition for the existence of stationary solitons in the model. It is necessary to check if this condition is compatible with the other one, Eq. (4), which is necessary for the stability of solitons in the model. Because Eq. (4) does not involve the parameter $\gamma_{1}$, one can secure the compatibility, choosing small enough $\gamma_{1}$. If condition (8) is met, the solution with larger $\eta^{2}$ is stable, and the one with smaller $\eta^{2}$ is unstable, as solutions to Eq. (7). The unstable solution is a separatrix between the stable soliton and zero solution.

In order to check the analytical predictions, we solved Eqs. (2) and (3) numerically by means of the split-step Fourier method [7]. In the first runs of the simulations, the initial

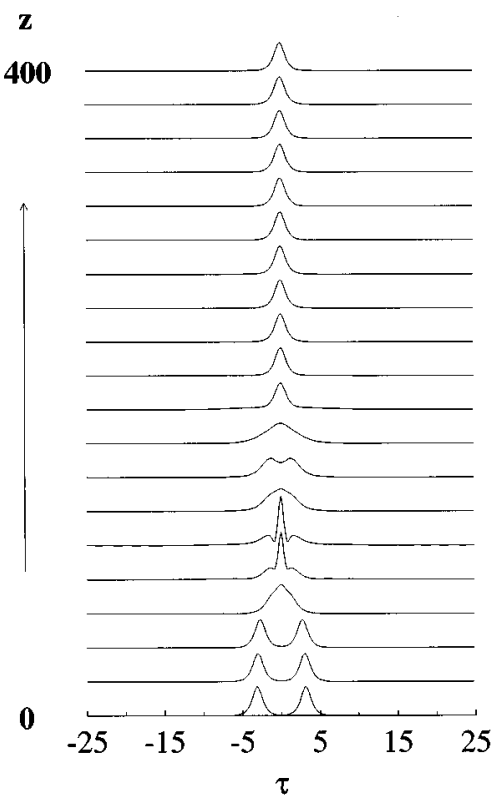

FIG. 2. The fusion of two in-phase pulses into a single pulse.

state was taken in the form of a perturbed pulse (5) with different values of $\eta$. In all the cases, we observed a rapid evolution of the initial pulse into a stable one whose shape did not depend on the initial value of $\eta$. We will display results for a particular set of the values $\gamma_{0}=0.01, \gamma_{1}=0.01, \Gamma_{0}=0.15, \kappa=0.125$, which satisfy both conditions (4) and (8) necessary for the existence and stability of the pulses. Substitution of these values into Eq. (7) yields the stable fixed point at $\eta \approx 1.697$, which is expected to correspond to a stable pulse in Eqs. (2) and (3). A typical run of simulations is shown in Fig. 1. In order to test the stability of the pulse, we took initial conditions containing strong asymmetric disturbances. Measuring the amplitude of the established stable pulse in Fig. 1, we have found it to be $\approx 1.657$, which compares very well with the above analytical prediction 1.697. We tried, for comparison, to juxtapose the established pulse in Fig. 1 and the analytically predicted one. However, they proved to be virtually indistinguishable. These and many other runs of the simulations of Eqs. (2) and (3) produce cogent proof of the existence of the stable pulse in this model, with a shape very close to that predicted by the analytical perturbation theory.

Once stable solitary pulses have been found, the next step is to consider interactions between them. Since our pulses are close to the NLS solitons, one can expect that their interaction will be similar to the interaction between the solitons. As is well known, the latter interaction strongly depends on the relative phase $\psi$ of the solitons: it is attractive if $\psi=0$, and repulsive if $\psi=\pi$.

All the simulations to be displayed below were done for the same parameter values as above. We simulated the interaction between two pulses at $\psi=0$, taking at $z=0$ a superposition of two identical solitons with a certain initial separation $T_{\text {in }}$. Two different outcomes were observed, depending upon $T_{\text {in }}$ : fusion of the two pulses into a single one (Fig. 2), if the initial separation was not too large, and no interaction at all in the opposite case.

In the case where the fusion takes place, it produces a 


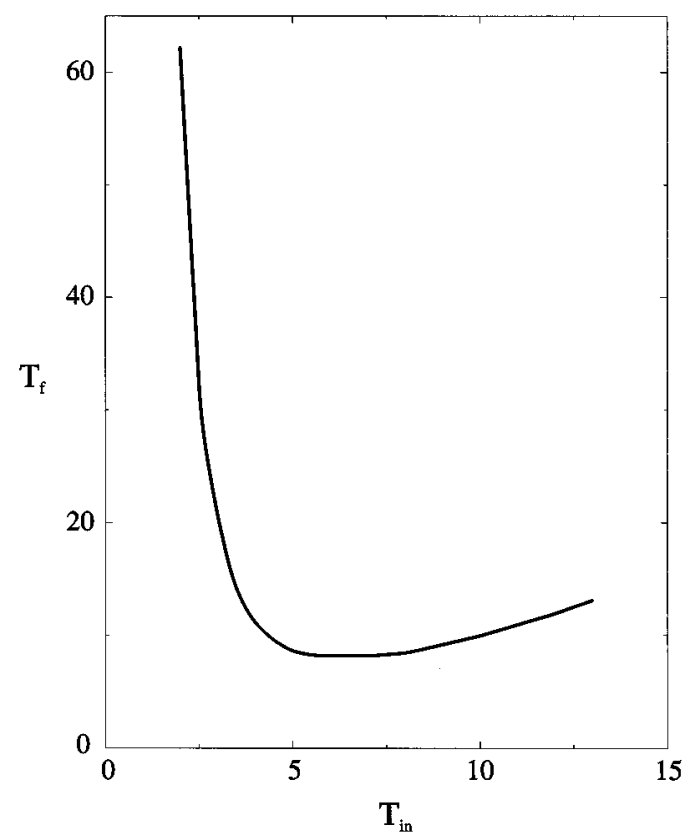

FIG. 3. The final separation between two $\pi$-out-of-phase pulses, measured at $z=400$, vs the initial separation between them.

stationary pulse which is identical to that generated by a solitary initial pulse, cf. Figs. 1 and 2. The simulations of the fusion provide another strong argument in favor of robustness of the stationary pulses in the model: as one can see in Fig. 2, the final pulse is generated from an intermediate "lump" which is very different from the stationary soliton.

One should expect repulsion between the pulses if the phase difference between them is $\pi$. In this case, we indeed observed repulsion. As a characteristic of this type of the interaction, we have plotted in Fig. 3 the final separation between the solitons, $T_{f}$, versus the initial separation $T_{\text {in }}$. This plot pertains to the same parameter values as above. As can be seen, the strong repulsion between initially close pulses pushes them far apart. Then, $T_{f}$ decreases with the increase of the $T_{\text {in }}$ due to the weakening repulsion. A minimum of $T_{f}$ is attained when $T_{\text {in }}$ takes values between 6.0 and 7.0: in this interval, $T_{f}$ remains practically constant, equal to $T_{\min }=8.203$. With further increase of $Y_{\mathrm{in}}, T_{f}$ is increasing, and at $T_{\mathrm{in}}>9.5$ the dependence becomes trivial, $T_{f} \approx T_{\mathrm{in}}$, which simply implies the absence of any conspicuous interaction.

The flat minimum at $T_{f}=T_{\min }$ in the dependence shown in Fig. 3 looks like a bound state of the solitons. Indeed, a bound state implies that the final separation takes a fixed value independent of the initial separation [4]. However, in the present case the flat minimum is not a true bound state. This is suggested by the fact that the value $T_{f} \approx 8.58$ corresponding to $T_{\mathrm{in}}=T_{\min }=8.203$ is different from $T_{\min }$ itself. The final separation $T_{f}$ shown in Fig. 3 is measured at $z=400$. We have checked that at $z=2000$ the results are practically the same.

We will call the feature displayed in Fig. 3 a pseudobound state. Although this state is not truly bound, it may play a significant role in applications, as it looks like a bound state, is robust, and is observed at moderate separations. True bound states of solitons in the present model were considered

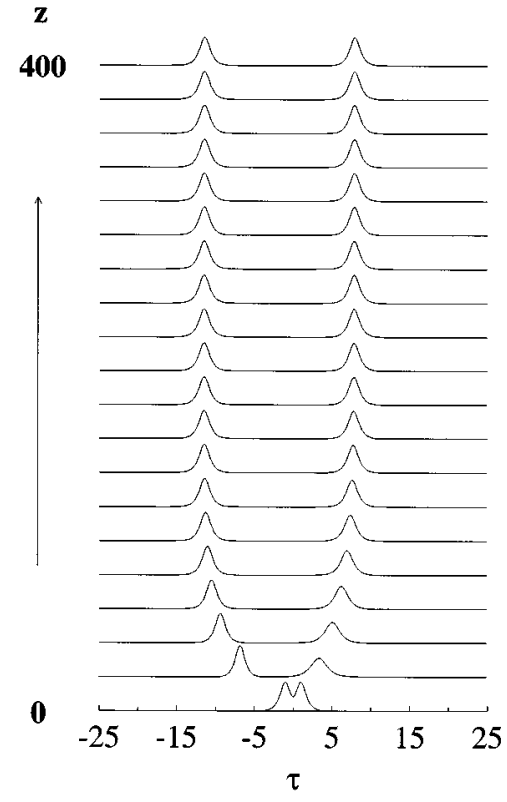

FIG. 4. Repulsion between two pulses with the initial phase difference $\pi / 2$.

in [5]. They may exist because the linear terms accounting for the gain and dissipation render the solitons' tails oscillatory, which, in turn, gives rise to local minima in the effective potential of the soliton-soliton interaction. In Ref. [5], it was shown that the minimum separation between the solitons in the true bound state is $T \approx \pi \eta / \Gamma_{0}$. Substitution of the present parameter values yields $T \approx 36$, which is much larger than the above $T_{\min }$. Actually, at such a large separation there is no interaction between the solitons. A true bound state can be observed at larger values of the perturbation parameters, but we will not consider this issue.

The pseudo-bound state can be explained by means of the perturbation theory which treats interaction between the solitons as a small perturbation [1]. It is straightforward to derive an evolution equation for the separation $T(z)$ between two identical solitons:

$$
\ddot{T}=-8 \eta^{3} \cos \psi e^{-\eta T}-\gamma_{1} \dot{T}\left[2 \eta^{2}+\frac{3}{2} \dot{T}^{2}\right],
$$

the overdot standing for $d /(d z)$. Consideration of Eq. (9) with $\cos \psi=-1$ (repulsion) shows that the dependence of the final separation on the initial separation will indeed have a minimum, provided that the dissipative constant $\gamma_{1}$ is small enough. Indeed, looking at Eq. (9) as an equation of motion for a unit-mass particle with the coordinate $T(z)$ in the potential $U(T) \equiv 8 \eta^{2} \exp (-\eta T)$, one concludes that, neglecting the friction force, the particle with the initial position $T_{\text {in }}$ will acquire at $z \rightarrow \infty$ the "velocity" $\dot{T}_{\infty}=4 \eta \exp \left(-\frac{1}{2} \eta T_{\text {in }}\right)$. Now, we take into account that the moving particle is braked by the weak friction force according to the equation $\ddot{T}=-2 \gamma_{1} \eta^{2} \dot{T}$, which yields the total "distance" traveled by the particle, $T_{f} \equiv T(z=\infty)=\left(2 \gamma_{1} \eta^{2}\right)^{-1} \dot{T}_{\infty}$. Finally, inserting $\dot{T}_{\infty}$ into the latter expression, one obtains 


$$
T_{f}=2\left(\gamma_{1} \eta\right)^{-1} \exp \left(-\frac{1}{2} \eta T_{\text {in }}\right),
$$

which is indeed a decreasing function of the initial separation $T_{\text {in }}$. On the other hand, at very large $T_{\text {in }}$ the particle will not feel the exponentially weak interaction force, hence one will trivially have $T_{f} \approx T_{\text {in }}$. Thus, there must be a minimum of $T_{f}$ at some intermediate value of $T_{\text {in }}$.

We have also considered the case with the initial phase difference $\psi=\pi / 2$, when the formal perturbation theory predicts zero interaction force [see Eq. (9)]. In this case, too, we have observed repulsion, but with a broken symmetry be-

[1] Yu.S. Kivshar and B.A. Malomed, Rev. Mod. Phys. 61, 763 (1989).

[2] A. Hasegawa and Y. Kodama, Solitons in Optical Communications (Oxford University Press, Oxford, 1995).

[3] L.M. Hocking and K. Stewartson, Proc. R. Soc. London, Ser. A 326, 289 (1972); N.R. Pereira and L. Stenflo, Phys. Fluids 20, 1733 (1977). tween the two solitons (Fig. 4). It is easy to understand this symmetry breaking. Indeed, if $\psi$ is 0 or $\pi$, the initial configuration at $z=0$ is the linear superposition $u(\tau-$ $\left.\frac{1}{2} T_{\text {in }}\right) \pm u\left(\tau+\frac{1}{2} T_{\text {in }}\right)$, where $u(\tau)$ is given by Eq. (5). Obviously, the solution generated by this initial configuration will keep the symmetry property $u(-\tau)= \pm u(\tau)$ at any $z>0$. In the case $\psi=\pi / 2$, the initial state is $u\left(\tau-\frac{1}{2} T_{\text {in }}\right)+i u\left(\tau+\frac{1}{2}\right.$ $\left.T_{\text {in }}\right)$. This configuration possesses only the symmetry $u(-\tau)=i u^{*}(\tau)$, which is not compatible with Eqs. (2) and (3), and is therefore broken by the evolution.

We thank J. M. Soto-Crespo for useful discussions of numerical methods.
[4] B.A. Malomed, Phys. Rev. A 44, 6954 (1991); Phys. Rev. E 47, 2874 (1993).

[5] B.A. Malomed and H.G. Winful, Phys. Rev. E 53, 5365 (1996).

[6] H.G. Winful and D.T. Walton, Opt. Lett. 17, 1688 (1992).

[7] M.D. Feit and J.A. Fleck, Jr., Appl. Opt. 17, 3990 (1978); S.L. Doty, J.W. Haus, Y.J. Oh, and R.L. Fork, Phys. Rev. E 51, 709 (1995). 